\title{
Dirichlet Forms and White Noise Analysis
}

T. Hida ${ }^{1}$, J. Potthoff ${ }^{2}$, and L. Streit ${ }^{3}$

${ }^{1}$ Department of Mathematics, Nagoya University

${ }^{2}$ Fachbereich Mathematik, Technische Universität Berlin, D-1000 Berlin 12

${ }^{3}$ BiBoS, Universität Bielefeld

\begin{abstract}
We use the white noise calculus as a framework for the introduction of Dirichlet forms in infinite dimensions. In particular energy forms associated with positive generalized white noise functionals are considered and we prove criteria for their closability. If the forms are closable, we show that their closures are Markovian (in the sense of Fukushima).
\end{abstract}

\section{Introduction}

In the past decade the theory of Dirichlet forms [5] has become an increasingly important link between probability theory, analysis, quantum theory and stochastic mechanics [1-6]. The infinite dimensional case is of particular interest for the development of infinite dimensional analysis and of quantum and stochastic models with infinitely many degrees of freedom $[1,14,19,20]$.

Here white noise analysis $[7-9,11,13]$ turn out to offer a particularly suitable framework; the present article is just a first step towards the filling out of this frame.

In Sect. 2 we assemble some pertinent material from white noise analysis, in particular concerning positive white noise functionals and their representation by measures. As in the finite dimensional case the underlying nuclear rigging is far from unique, and different alternatives should be explored, with a view towards different applications.

In Sect. 3 we construct energy forms from positive generalized functionals of white noise. We give criteria for the admissibility of these functionals, so that the forms correspond to positive self-adjoint operators, generalizing the generator of the infinite dimensional Ornstein-Uhlenbeck process. We show that the construction goes beyond the case of measures which are absolutely continuous with respect to white noise; i.e. much wider classes of sample functions are allowed to occur. Finally we demonstrate the Markov property for the forms that we construct and conclude the paper by commenting on the Markov processes, which are generated by the Markovian semigroups associated with our forms. 
For simplicity of our exposition, we have written this article using the basic nuclear Gel'fand triple,

$$
\mathscr{S}^{*}(\mathbb{R}) \curvearrowright L^{2}(\mathbb{R}, d t) \curvearrowright \mathscr{S}(\mathbb{R}),
$$

which generates the probability space $\left(\mathscr{S}^{*}(\mathbb{R}), \mathscr{B}, d \mu\right)$ of white noise with one dimensional time $[7,8]$. However, our constructions and results are readily generalized to any Gel'fand triple of function spaces of the type (1.1). The class of the associated Gaussian probability spaces will then for example include white noise with higher dimensional time, Euclidean quantum field theory and so forth.

\section{Generalized White Noise Functionals and Measures}

To generalize the concept of white noise functionals it is convenient to embed the $L^{2}$-space over the white noise probability space in a Gel'fand triple of smooth, respectively generalized functionals. Such a construction is far from unique and should be adapted to the case at hand. In the following we construct a particular example of such a triple, the properties of which are convenient for our purpose.

Let $(\mathscr{S} *(\mathbb{R}), \mathscr{B}, d \mu)$ be the probability space of white noise $(\mathrm{cf} .[7,8])$ and denote

$$
\left(L^{2}\right):=L^{2}(\mathscr{S} *(\mathbb{R}), \mathscr{B}, d \mu) .
$$

Recall the correspondence between white noise functionals $F$ and sequences $\left(F_{n}, n \in \mathbb{N}_{0}\right)$ of symmetric square integrable kernel functions $F_{n} \in \overline{L^{2}\left(\mathbb{R}^{n}\right)}\left(F_{0} \in \mathbb{C}\right)$ given by

$$
\left(L^{2}\right) \simeq \bigoplus_{n \in \mathbb{N}_{0}} L^{2}\left(\widehat{\mathbb{R}^{n}}, n ! d^{n} t\right)
$$

which is the standard isometry between $\left(L^{2}\right)$ and the symmetric Fock space over $L^{2}(\mathbb{R}, d t)[7,8,13,18]$. It is convenient to implement $(2.2)$ by the following transformation:

$$
(S F)(\xi):=\int F(x+\xi) d \mu(x)
$$

for $F \in\left(L^{2}\right)$ and $\xi \in \mathscr{S}(\mathbb{R})[11,13]$. If $F \in\left(L^{2}\right)$ corresponds to $\left(F_{n}\right)$ in the above sense, then

$$
(S F)(\xi)=\sum_{n=0}^{\infty} \int_{\mathbb{R}^{n}} F_{n}\left(t_{1}, \ldots, t_{n}\right) \xi\left(t_{1}\right) \ldots \xi\left(t_{n}\right) d^{n} t
$$

Now let $A$ be a densely defined linear operator on $L^{2}(\mathbb{R}, d t)$. Then there is its "second quantized" operator [18]

$$
\Gamma(A)=\bigoplus_{n} A^{\otimes n}
$$

acting on the Fock space on the right-hand side of (2.2), and this way we are given an operator $S^{-1} \Gamma(A) S$ on $\left(L^{2}\right)$. For simplicity we shall denote this operator too by $\Gamma(A)$. Note that $\Gamma(A)$ is densely defined, linear and - on appropriate domains $\Gamma(A)^{p}=\Gamma\left(A^{p}\right), p \in \mathbb{N}$.

In particular we consider

$$
A=1+t^{2}-\frac{d^{2}}{d t^{2}}
$$


with Hermite eigenfunctions $e_{k}, k \in \mathbb{N}_{0}$, and $A e_{k}=(2 k+2) e_{k}$. Denoting

$$
\left(\mathscr{S}_{p}\right):=\mathscr{D}\left(\Gamma\left(A^{p}\right)\right) \subset\left(L^{2}\right)
$$

we obtain a chain of continuously and densely embedded Hilbert spaces $(p \in \mathbb{N})$

$$
\ldots \subset\left(\mathscr{S}_{p+1}\right) \subset\left(\mathscr{S}_{p}\right) \subset \ldots \subset\left(L^{2}\right) \subset \ldots \subset\left(\mathscr{S}_{-p}\right) \subset\left(\mathscr{P}_{-p-1}\right) \subset \ldots
$$

whose scalar products, respectively norms we denote by $(\cdot, \cdot)_{2, p}$ and $\|\cdot\|_{2, p}$. It is easy to see that the system $\left((\cdot, \cdot)_{2, p} ; p \in \mathbb{Z}\right)$ is compatible. Now we define the space $(\mathscr{P})$ of white noise test functionals as the projective limit of the chain (2.8), i.e.

$$
(\mathscr{P})=\bigcap_{p}\left(\mathscr{S}_{p}\right)
$$

and $(\mathscr{S})$ is provided with the projective limit topology. $(\mathscr{S})$ is countable Hilbert and therefore its dual $(\mathscr{P})^{*}$ is given by

$$
(\mathscr{P})^{*}=\bigcup_{p}\left(\mathscr{S}_{-p}\right)
$$

since $\left(\mathscr{S}_{p}\right)^{*}=\left(\mathscr{S}_{-p}\right)$. By choice of $A(\mathscr{P})$ is nuclear. As usual we shall say that " $\Phi \in(\mathscr{S})^{*}$ has order $p$ ", if $p$ is the minimal element in $\mathbb{N}_{0}$ such that $\Phi \in\left(\mathscr{S}_{-p}\right)$.

Let $\xi \in \mathscr{S}(\mathbb{R})$ and consider the $\mathscr{S}^{*}(\mathbb{R})$-functional

$$
F(x)=e^{i\langle x, \xi\rangle}, \quad x \in \mathscr{S}^{*}(\mathbb{R}) .
$$

Then

$$
\Gamma\left(A^{p}\right) F(x)=e^{i\left\langle x, A^{p} \xi\right\rangle+\frac{1}{2}\left(\xi,\left(A^{p}-1\right) \xi\right)},
$$

and hence the algebra $\mathscr{E}$ generated by the functionals of the form $(2.11)$ is contained in $(\mathscr{S})$. By a result in [8] we therefore have

Lemma 2.1. $(\mathscr{S})$ is dense in $\left(L^{2}\right)$. lemma:

Another important property of this test functional space is the following

Lemma $2.2[11] .(\mathscr{P})$ is an algebra.

The somewhat technical proof of this lemma is deferred to an appendix.

For $x \in \mathscr{S}^{*}(\mathbb{R})$ we define the normal ordered product (in a slightly informal notation)

$$
: x^{\otimes n}:\left(t_{1}, \ldots, t_{n}\right) \equiv: x\left(t_{1}\right) \ldots x\left(t_{n}\right):
$$

in $\mathscr{S} *\left(\mathbb{R}^{n}\right)$ with respect to the (informal) covariance

$$
\mathbb{E}\left(x\left(t_{1}\right) x\left(t_{2}\right)\right)=\delta\left(t_{1}-t_{2}\right),
$$

so that e.g. : $x\left(t_{1}\right) x\left(t_{2}\right):=\delta\left(t_{1}-t_{2}\right)$ (cf. also [18]).

Lemma 2.3 [12]. Each $F \in(\mathscr{S})$ has a version $\tilde{F}$ of the form

$$
\tilde{F}(x)=\sum_{n}\left\langle: x^{\otimes n}:, F_{n}\right\rangle
$$


with $F_{n}$ in $\overline{\mathscr{S}\left(\mathbb{R}^{n}\right)}$. Conversely any such $\tilde{F}$ is in $(\mathscr{S})$ iff

$$
\sum_{n} n !\left\|\Gamma\left(A^{p}\right) F_{n}\right\|_{2}^{2}<\infty
$$

for all $p \in \mathbb{N}_{0}$.

Let $(\mathscr{S})_{0}$ denote the subspace of $(\mathscr{S})$ consisting of those $F$ which have only a finite number of nonvanishing $F_{n}\left[\mathrm{cf}\right.$. (2.13)]. Clearly $(\mathscr{S})_{0}$ is dense in all $\left(\mathscr{S}_{p}\right), p \in \mathbb{Z}$. For all $F \in(\mathscr{S})_{0}$ and all $f \in L^{2}(\mathbb{R}, d t)$ the Fréchet derivative $D_{f}$ of $S F$ [cf. (2.4)] is well-defined. In fact, viewed as an operator on Fock space $D_{f}$ is nothing but the annihilation operator of $f$ [18]. The closure of this operator in Fock space is denoted by the same symbol and we set

$$
\partial_{f}:=S^{-1} D_{f} S \text {. }
$$

If in particular $f=e_{k}, k \in \mathbb{N}_{0}$, we simply write $\partial_{k}$. We have the following

Lemma 2.4. For all $f \in L^{2}(\mathbb{R}, d t)$,

$$
\partial_{f}:(\mathscr{S}) \rightarrow(\mathscr{S}) .
$$

Proof. By elementary calculation one derives the bound

$$
\left\|\partial_{f} F^{(n)}\right\|_{2, p} \leqq n^{1 / 2} 2^{-n}\left\|A^{-1} f\right\|_{2}\left\|F^{(n)}\right\|_{2, p+1}
$$

for $F^{(n)}$ given by

$$
F^{(n)}(x)=\left\langle: x^{\otimes n}:, F_{n}\right\rangle
$$

$F_{n} \in \overline{\mathscr{S}\left(\mathbb{R}^{n}\right)}$. Since $F \in(\mathscr{S})$ is given by a sum of such functionals satisfying (2.14), it is clear by Lemma 2.3 that the estimate $(2.17)$ proves the assertion.

Let us denote

$$
\left(\mathscr{L}^{2}\right):=\left(L^{2}\right) \otimes l^{2}
$$

$l^{2}$ being the Hilbert space of square summable sequences over $\mathbb{N}_{0}$. We introduce

$$
\nabla:\left(L^{2}\right) \rightarrow\left(\mathscr{L}^{2}\right)
$$

by

$$
\nabla F:=\left(\partial_{0} F, \partial_{1} F, \ldots, \partial_{k} F, \ldots\right),
$$

defined on $(\mathscr{S})_{0}$. It is easy to see that $\nabla$ is closable. Its closure is denoted by the same symbol. Also we set

$$
|\nabla F|^{2}:=\sum_{k \in \mathbb{N}_{0}}\left|\partial_{k} F\right|^{2}
$$

Next we prove the following

Lemma 2.5. $F \in(\mathscr{S})$ entails $|\nabla F|^{2} \in(\mathscr{S})$.

Proof. Let $p \in \mathbb{N}_{0}$. Then

$$
\left\||\nabla F|^{2}\right\|_{2, p} \leqq \sum_{k, m, n}\left\|\left(\partial_{k} \bar{F}^{(m)}\right)\left(\partial_{k} F^{(n)}\right)\right\|_{2, p},
$$


and by the estimate in the proof of Lemma 2.2 in the appendix we know that there is $q \in \mathbb{N}$ and $c_{q}>0$ so that

$$
\left\||\nabla F|^{2}\right\|_{2, p} \leqq c_{q} \sum_{k, m, n}\left\|\partial_{k} F^{(m)}\right\|_{2, p+q}\left\|\partial_{k} F^{(n)}\right\|_{2, p+q} .
$$

Now apply inequality (2.17), use $A e_{k}=(2 k+2) e_{k}$ and Schwarz' inequality to conclude the proof.

Next we want to summarize results [21], cf. also [15], about positive generalized functionals and measures.

Definition 2.6. We introduce the cone of positive test functionals as

$$
(\mathscr{S})_{+}:=\{F \in(\mathscr{S}): F \geqq 0 \mu \text {-a.e. }\} \text {. }
$$

and call $\Phi \in(\mathscr{S})^{*}$ a positive generalized functional if it maps $(\mathscr{S})_{+}$into the positive real numbers.

Theorem 2.7 [21]. For any positive generalized functional $\Phi$ there is a unique positive finite measure $v$ on the Borel algebra over $\mathscr{S}^{*}(\mathbb{R})$ such that for all $F \in(\mathscr{S})$,

$$
\langle\Phi, F\rangle=\int \tilde{F} d v
$$

Remark. $\Phi$ plays, as in finite dimensional analysis, the role of a (possible generalized) Radon-Nikodym derivative, and here as there the notation $d v=\Phi d \mu$ should not mean to imply absolute continuity. For short we shall write $\left(L^{2}\right)_{v}$ for $L^{2}(\Phi d \mu)$, and similarly $\left(\mathscr{L}^{2}\right)_{v}$.

Example 2.8. Consider any strictly positive operator $\tilde{K}$ on $L^{2}(\mathbb{R}, d t)$ which obeys

$$
\left\|A^{-p}(\tilde{K}-1) A^{-p}\right\|_{\text {H.S. }}<1, \quad\left\|A^{-p} \tilde{K}^{2} A^{-p}\right\|_{\text {H.S. }}<\infty .
$$

Then

$$
\int e^{i\langle x, \xi\rangle} \Phi(x) d \mu(x)=\exp (-1 / 2(\xi, \tilde{K} \xi))
$$

defines a positive generalized functional $\Phi$ of order $p$. We shall also use the notation

$$
\Phi(x)=N \exp (-1 / 2(x, K x))
$$

with $K=\widetilde{K}^{-1}(1-\tilde{K})$, generalizing from the case of trace class $K$, for which the $N$ indicates division by the expectation.

\section{Dirichlet Forms from Positive White Noise Functionals}

In this section we shall study "energy forms" generated by positive generalized white noise functionals $\Phi$. For clarity of our exposition we shall focus on forms given by

$$
\varepsilon(F) \equiv\left\langle\Phi,|\nabla F|^{2}\right\rangle=\int|\nabla F|^{2} d \nu,
$$

leaving more general expressions such as e.g.

$$
\left\langle\Phi, \sum_{i k} G^{i k}\left(\partial_{i} \bar{F}\right)\left(\partial_{k} F\right)\right\rangle+\left\langle\Phi, H F^{2}\right\rangle
$$

for a separate investigation. 
Theorem 3.1. The energy form $\varepsilon$ arising from a positive generalized white noise functional $\Phi$, defined on $(\mathscr{S})$, is a positive, densely defined, symmetric quadratic form on $\left(L^{2}\right)_{v}$.

Proof. Note that by Lemma 2.2 and Theorem 2.7,

$$
\mathscr{D}(\varepsilon)=(\mathscr{S}) \subset\left(L^{2}\right)_{v} .
$$

Furthermore, the algebra generated by functionals of the form (2.11) is dense in $\left(L^{2}\right)_{y}$ (by a Stone-Weierstraß argument) and is contained in $(\mathscr{S})$, so that the embedding (3.3) is dense. The other assertions are obvious.

We now turn to the problem of closability of the quadratic form $\varepsilon$.

Definition 3.2. A positive generalized functional $\Phi$ is called admissible if the corresponding energy form (3.1) is closable on $\left(L^{2}\right)_{v}$.

As a consequence we have the following

Theorem 3.3. Let $\Phi$ be admissible. $\bar{\varepsilon}$ denotes the closure of $\varepsilon, \mathscr{D}(\bar{\varepsilon})$ its domain. Then there exists a unique positive self-adjoint operator $H$ with $\mathscr{D}\left(H^{1 / 2}\right)=\mathscr{D}(\bar{\varepsilon})$ and such that $\varepsilon(F)=\left\|H^{1 / 2} F\right\|_{\left(L^{2}\right)_{v}}^{2}$.

This is a direct consequence of general theorems given in [10]. The same is true for the following

Theorem 3.4. $\varepsilon$ is closable if

$$
\nabla^{*}:\left(\mathscr{L}^{2}\right)_{v} \rightarrow\left(L^{2}\right)_{v}
$$

is densely defined.

Example 3.5. $\Phi=1$ : in this case $-H$ is the Laplace-Beltrami operator

$$
H=\sum_{k} \partial_{k}^{*} \partial_{k}
$$

where $\partial_{k}^{*}$ is the $\left(L^{2}\right)$-adjoint of $\partial_{k}$. $H$ is of course the $S$-transform of the number operator on Fock space and generates the infinite-dimensional OrnsteinUhlenbeck process on $\mathscr{S}^{*}(\mathbb{R})[14,19]$.

In order to describe a convenient criterion in the case that $v$ is absolutely continuous with respect to $\mu$ with positive density $\Phi$, we introduce the spaces $\left(L^{p, q}\right)$, $p=2,3, \ldots, q \in \mathbb{N}_{0}$, which are the completions of the algebra $\mathscr{E}$ generated by functionals of the form (2.11) under the norms

$$
\|\mathrm{F}\|_{p, q}:=\left\|(1+H)^{q} F\right\|_{p},
$$

where $H$ is the number operator (3.5). Note that $(\mathscr{S}) \subset\left(L^{p . q}\right) \subset\left(L^{2}\right)$ for all $p=2,3, \ldots, q \in \mathbb{N}_{0}$ and that these embeddings are dense and continuous.

Theorem 3.6. Assume that $\Phi>0$ ( $\mu$-a.e.) and $\Phi^{1 / 2} \in\left(L^{4,1}\right)$. Then $\Phi$ is admissible.

Remark. The condition on $\Phi$ in this theorem is not optimal, but it allows for a transparent proof. However, note that our condition is less restrictive than the one used in [19].

The proof of Theorem 3.6 makes use of the following 
Lemma 3.7. Under the hypothesis of Theorem 3.6,

$$
\partial_{k} \Phi^{1 / 2}=1 / 2 \Phi^{1 / 2} \partial_{k} \Phi
$$

for all $k \in \mathbb{N}_{0}$ in $\left(L^{2}\right)$-sense.

Proof. From the assumptions it follows that there is a sequence $\left(f_{n} ; n \in \mathbb{N}\right)$ in $\mathscr{E}$, so that

$$
f_{n} \rightarrow \Phi^{1 / 2} \quad \text { in }\left(L^{4,1}\right)
$$

and

$$
f_{n}^{2} \rightarrow \Phi \quad \text { in }\left(L^{2}\right)
$$

It is easy to check that for every $k \in \mathbb{N}_{0} \partial_{k}$ is a derivation on $\mathscr{E}$, so that for every $k \in \mathbb{N}_{0} \partial_{k} f_{n}^{2}$ is Cauchy in $\left(L^{2}\right)$. Moreover $\partial_{k} \Phi$ is in $\left(L^{2}\right)$, which can be seen as follows:

$$
\left\|\partial_{k} \Phi\right\|_{2} \leqq\||\nabla \Phi|\|_{2}=\left\|H^{1 / 2} \Phi\right\|_{2} \leqq\|H \Phi\|_{2} \leqq \text { const }\left\|H \Phi^{1 / 2}\right\|_{4}^{2},
$$

where the last inequality followed from Meyer's equivalence, cf. e.g. [17]. Thus the closed graph theorem implies that

$$
\partial_{k} \Phi=\partial_{k}\left(\Phi^{1 / 2}\right)^{2}=\lim _{n} \partial_{k} f_{n}^{2}=2 \lim _{n} f_{n} \partial_{k} f_{n}=2 \Phi^{1 / 2} \partial_{k} \Phi^{1 / 2},
$$

where the limits are taken in $\left(L^{2}\right)$.

Proof of Theorem 3.6. Let $\left(F_{k} ; k \in \mathbb{N}_{0}\right)$ be a sequence with $F_{k}=0$ for almost all $k \in \mathbb{N}_{0}$ and $F_{k} \in \mathscr{E}$ for all $k \in \mathbb{N}_{0}$. Then $\mathbf{F} \equiv\left(F_{k}\right) \in\left(\mathscr{L}^{2}\right)_{v}$ and

$$
\nabla^{*} \mathbf{F}=\sum_{k}\left(-\partial_{k}+x_{k}-\left(\Phi^{-1} \partial_{k} \Phi\right)\right) F_{k}
$$

where $x_{k}$ is the multiplication operator $\left(x_{k} F\right)(x)=\left\langle x, e_{k}\right\rangle F(x)$. Thus

$$
\begin{aligned}
\left\|\nabla^{*} \mathbf{F}\right\|_{\left(L^{2}\right)_{v}}^{2} \leqq & 2\left\{\int d \mu(x) \Phi(x)\left|\sum_{k}\left(-\partial_{k}+x_{k}\right) F_{k}(x)\right|^{2}\right. \\
& \left.+\int d \mu(x) \Phi(x)\left|\sum_{k}\left(\Phi^{-1} \partial_{k} \Phi\right) F_{k}(x)\right|^{2}\right\} .
\end{aligned}
$$

Since $F_{k} \in \mathscr{E}$ and $\Phi \in\left(L^{2}\right)$, it is a trivial application of Schwarz' inequality to show that the first of the last two terms is bounded. For the second term note that each $F_{k}$ is bounded and that the sum has only a finite number of terms. Thus it suffices to show that

$$
\sum_{k} \int d \mu(x) \Phi(x)\left(\Phi^{-1} \partial_{k} \Phi\right)^{2}(x)
$$

is bounded. By Lemma 3.7 this expression equals

$$
4 \int d \mu(x)\left|\nabla \Phi^{1 / 2}\right|^{2}(x)=4\left\|H^{1 / 2} \Phi^{1 / 2}\right\|_{2}^{2},
$$

which is finite by our assumption.

In the case that $\Phi$ is a genuine generalized functional, i.e. $v$ not absolutely continuous with respect to $\mu$, we have the following criterion.

Theorem 3.8. $\Phi$ is admissible if $\partial_{k} \Phi=B_{k} \Phi$ with $B_{k} \in(\mathscr{S})$ for every $k \in \mathbb{N}_{0}$. 
Remark. Here we mean by $\partial_{k} \Phi$ for $\Phi \in(\mathscr{S})^{*}$ the distributional derivative

$$
\left\langle\partial_{k} \Phi, F\right\rangle:=\left\langle\Phi, \partial_{k}^{*} F\right\rangle, \quad F \in(\mathscr{S}),
$$

which exists to all orders.

Proof. Let $G=\left(G_{k} ; k \in \mathbb{N}_{0}\right) \in\left(\mathscr{L}^{2}\right)_{v}$, i.e.

Then $\nabla^{*}$ acts as

$$
\sum_{k}\left\|G_{k}\right\|_{\left(L^{2}\right)_{v}}^{2}<\infty \text {. }
$$

$$
\nabla^{*} G=\sum_{k}\left(-\partial_{k}+x_{k}-B_{k}\right) G_{k}
$$

Thus $\nabla^{*}$ is well-defined on the space of those sequences $G$ in $\left(\mathscr{L}^{2}\right)_{v}$ which have only a finite number of zero entries from $(\mathscr{S})$. This space is clearly dense in $\left(\mathscr{L}^{2}\right)_{v}$.

Other, more general, conditions may be thought of, but the above already suffices to demonstrate that the class of admissible generalized functionals and hence our construction of energy forms is not restricted to $\Phi \in L^{1}(d \mu)$, i.e. to absolutely continuous measures $v<\mu$.

Example 3.9. Consider (cf. Example 2.8)

for which one finds

$$
\Phi=: \exp (-1 / 2(x, K x)):
$$

$$
B_{k}(x)=-(K x)_{k}=-\left\langle x, K e_{k}\right\rangle,
$$

which is in $(\mathscr{S})$ if $K$ maps $\mathscr{S}(\mathbb{R})$ into itself.

As a next step in the development of our subject we would like to investigate the Markov property [5] of the energy forms which we have constructed.

Theorem 3.10. Let $\Phi$ be an admissible positive generalized functional and $\bar{\varepsilon}$ be the closure of the associated energy form $\varepsilon$. Then $\bar{\varepsilon}$ is Markovian in the sense of Fukushima [5].

Proof. By $\mathscr{A}$ we mean the algebra of functionals generated by $\sin \langle\cdot, \xi\rangle, \cos \langle\cdot, \xi\rangle$, $\xi \in \mathscr{S}(\mathbb{R})$. Using a Weierstraß argument we find that the composite $g \circ F$ of a functional $F \in \mathscr{A}$ with a $C^{1}(\mathbb{R})$-function $g$ is in $\mathscr{D}(\vec{\varepsilon})$. Moreover note that $\mathscr{A}$ is dense in $\mathscr{D}(\vec{\varepsilon})$. Now let $F \in \mathscr{D}(\vec{\varepsilon}), g \in C_{b}^{1}$, and $\left(F_{n} ; n \in \mathbb{N}\right)$ be a sequence in $\mathscr{A}$ approximating $F$ in $\mathscr{D}(\vec{\varepsilon})$, i.e. $F_{n} \rightarrow F$ in $\left(L^{2}\right)_{v}$ and $\bar{\varepsilon}\left(F-F_{n}\right) \rightarrow 0$. Selecting a subsequence, we may assume that $F_{n} \rightarrow F$ a.e. Since $v$ is finite and $g$ bounded, the dominated convergence theorem implies that $g \circ F_{n} \rightarrow g \circ F$ in $\left(L^{2}\right)_{v}$. Furthermore, by the chain rule [16]

$$
\begin{aligned}
\bar{\varepsilon}\left(g \circ F_{n}-g \circ F_{m}\right)= & \int\left|\left(g^{\prime} \circ F_{n}\right) \nabla F_{n}-\left(g^{\prime} \circ F_{m}\right) \nabla F_{m}\right|^{2} d v \\
\leqq & \left\|g^{\prime}\right\|_{\infty} \int\left|\nabla F_{n}-\nabla F_{m}\right|^{2} d v \\
& +\int\left|\nabla F_{n}\right|^{2}\left|g^{\prime} \circ F_{n}-g^{\prime} \circ F_{m}\right|^{2} d v .
\end{aligned}
$$

The first term vanishes since $\bar{\varepsilon}\left(F-F_{n}\right) \rightarrow 0$ and the second converges to zero by the dominated convergence theorem. Since $\bar{\varepsilon}$ is closed $g \circ F \in \mathscr{D}(\bar{\varepsilon})$.

Now let $\varphi_{\delta}, \delta>0$, be the smooth truncation defined in [2, Theorem 3.2], then (using the chain rule and closedness of $\bar{\varepsilon}$ )

for all $F \in \mathscr{D}(\tilde{\varepsilon})$.

$$
\bar{\varepsilon}\left(\varphi_{\delta} \circ F\right) \leqq \bar{\varepsilon}(F)
$$


We conclude this paper by some remarks concerning the processes associated with the forms we have constructed.

From Theorem 3.3, Theorem 3.10 and general theory [5] we know that an admissible positive generalized functional $\Phi \in\left(\mathscr{S}^{*}\right)$ defines a positivity preserving, strongly continuous contraction semigroup $\left(P_{t} ; t \in \mathbb{R}_{+}\right)$on $\left(L^{2}\right)_{v}$ with $P_{t} 1=1$ for all $t \in \mathbb{R}_{+}$, whose generator is $H=\nabla^{*} \nabla$. This semigroup extends to a positivity preserving, strongly continuous contraction semigroup on all $\left(L^{p}\right)_{v}, 1 \leqq p \leqq \infty$, [18].

Now note that $\left(\mathscr{S}^{*}(\mathbb{R}), d v\right)$ is regular in the sense of Albeverio and HoeghKrohn [1, Sect. 3], because $d v$, as constructed above, is the extension of a cylinder set measure on $\mathscr{B}\left(\mathscr{S}^{*}(\mathbb{R})\right)$. Hence the arguments in [1] apply to associate with $\left(P_{t} ; t \in \mathbb{R}_{+}\right)$a canonical time homogeneous Markov process $\left(X(t) ; t \in \mathbb{R}_{+}\right)$realized on path space $\left(\left(\mathscr{S}^{*}(\mathbb{R})\right)^{[0, \infty)}, d \omega\right)$. Here $d \omega$ is the extension of the cylinder set measure defined by the initial measure $d v$ and the transition probabilities given by $\left(P_{t} ; t \in \mathbb{R}_{+}\right)$.

\section{Appendix}

In this appendix we prove Lemma 2.2. Remark that this result had already been announced (in a slightly more general formulation) by Kubo and Takenaka in [11].

First we introduce some notation. We denote $L\left(\mathbb{R}^{n}, n ! d^{n} t\right)$ by $\Gamma^{(n)}$ and set $\mathscr{H}^{(n)}:=S^{-1} \Gamma^{(n)}$, so that

in the sense of Sect. (2).

$$
\left(L^{2}\right)=\bigoplus_{n=0}^{\infty} \mathscr{H}^{(n)}
$$

If $f \in \Gamma^{(n)}$ and $r \in \mathbb{R}^{k}, k \leqq n$, then we mean by $f(r ; \cdot)$ the corresponding element in $\Gamma^{(n-k)}$. If $f(r ; \cdot)$ is in the domain of $\Gamma(A) 1_{\Gamma^{(n-k)}}$, we write $\Gamma_{n-k}(A) f(r ; \cdot)$ for the element in $\Gamma^{(n-k)}$ which results from the application of $\Gamma(A) 1_{\Gamma^{(n-k)}}$ to $f(r ; \cdot)$.

Furthermore, in order to avoid cumbersome formulae, we shall use the (somewhat informal) notation (cf. Sect. 2):

$$
\left\langle: x^{\otimes n}:, f\right\rangle=\int f\left(t_{1}, \ldots, t_{n}\right): x\left(t_{1}\right) \ldots x\left(t_{n}\right): d^{n} t
$$

for an appropriate function $f$ of $n$ variables. Now let

be of the form

$$
\varphi \in(\mathscr{S})^{(n-m)}:=(\mathscr{S}) \cap \mathscr{H}^{(n-m)} \text { and } \quad \psi \in(\mathscr{S})^{(m)}
$$

$$
\begin{gathered}
\varphi(x)=\int_{\mathbb{R}^{n-m}} f\left(t_{1}, \ldots, t_{n-m}\right): x\left(t_{1}\right) \ldots x\left(t_{n-m}\right): d^{n-m} t, \\
\psi(x)=\int_{\mathbb{R}^{m}} g\left(t_{1}, \ldots, t_{m}\right): x\left(t_{1}\right) \ldots x\left(t_{m}\right): d^{m} t .
\end{gathered}
$$

It is then a straightforward, though tedious computation using the definition of the normal-ordered products to derive the following formula $(k \wedge l \equiv \min (k, l))$ :

$$
\begin{aligned}
(\varphi \psi)(x)= & \sum_{k=0}^{n-m \wedge m} k !\left(\begin{array}{c}
n-m \\
k
\end{array}\right)\left(\begin{array}{l}
m \\
k
\end{array}\right) \int_{\mathbb{R}^{n-k}}(f(r ; \cdot) \hat{\otimes} g(r ; \cdot))\left(t_{1}, \ldots, t_{n-2 k}\right) \\
& \times: x\left(t_{1}\right) \ldots x\left(t_{n-2 k}\right): d^{n-2 k} t d^{r} k,
\end{aligned}
$$


which is the decomposition of $\varphi \psi$ into its homogeneous components in the spaces $\mathscr{H}^{(n-2 k)}$.

Now we are ready for the proof of the following

Proposition A.1. Let $\varphi$ and $\psi$ be as above. There is a constant $K$, independent of $\varphi, \psi, n, m, p$, so that

$$
\|\varphi \psi\|_{2, p} \leqq K^{n}\|\varphi\|_{2, p}\|\psi\|_{2, p} .
$$

Proof. Since $\Gamma\left(A^{p}\right)=\Gamma(A)^{p}$, it suffices to consider $p=1$. Using formula (A.3) and (2.2) we find

$$
\begin{aligned}
\|\Gamma(A) \varphi \psi\|_{2}^{2}= & \sum_{k=0}^{n-m \wedge m} c(n, m, k) \\
& \times \int_{\mathbb{R}^{n-2 k}}\left|\int_{\mathbb{R}^{k}} \Gamma_{n-m-k}(A) f(r ; t) \hat{\otimes} \Gamma_{m-k}(A) g(r ; t) d^{k}\right|^{2} d^{n-2 k} t
\end{aligned}
$$

with $c(n, m, k)=(n-2 k) !\left[k !\left(\begin{array}{c}n-m \\ k\end{array}\right)\left(\begin{array}{c}m \\ k\end{array}\right)\right]^{2}$.

Using Schwarz' inequality and $\left\|A^{-1}\right\| \leqq 1$, we obtain the estimate

$$
\begin{aligned}
\|\Gamma(A) \varphi \psi\|_{2}^{2} & \leqq \sum_{k=0}^{n-m \wedge m} c(n, m, k)\|\Gamma(A) f\|_{L^{2}\left(\mathbb{R}^{n-m}\right)}^{2}\|\Gamma(A) g\|_{L^{2}\left(\mathbb{R}^{m}\right)}^{2} \\
& =\left(\sum_{k=0}^{n-m \wedge m} c(n, m, k)((n-m) !)^{-1}(m !)^{-1}\right)\|\Gamma(A) \varphi\|_{2}^{2}\|\Gamma(A) \psi\|_{2}^{2} .
\end{aligned}
$$

Thus it remains to show that the above sum is bounded by $K^{n}$. But this is easily done using $(n-2 k) !(k !)^{2} \leqq n !, \sum_{k=0}^{q}\left(\begin{array}{l}q \\ k\end{array}\right)^{2}=\left(\begin{array}{c}2 q \\ q\end{array}\right)$ and Stirling's theorem.

Now we can prove Lemma 2.2:

For $\varphi, \psi \in(\mathscr{S})$, let $\varphi^{(n)}, \psi^{(n)}$ denote their components in $(\mathscr{S})^{(n)}$ and set

$$
\varphi \psi(x)=\sum_{n=0}^{\infty} \sum_{m=0}^{n} \varphi^{(n-m)}(x) \psi^{(m)}(x) .
$$

Then we have to show that the sum (A.4) converges in ( $\mathscr{P})$, i.e. in every norm $\|\cdot\|_{2, p}$. But

$$
\begin{aligned}
\|\varphi \psi\|_{2, p}^{2} & \leqq\left(\sum_{n=0}^{\infty} \sum_{m=0}^{n}\left\|\varphi^{(n-m)} \psi^{(m)}\right\|_{2, p}\right)^{2} \\
& \leqq\left(\sum_{n=0}^{\infty} K^{n} \sum_{m=0}^{n}\left\|\varphi^{(n-m)}\right\|_{2, p}\left\|\psi^{(m)}\right\|_{2, p}\right)^{2} \\
& \leqq\left(\sum_{n=0}^{\infty} 2^{-n} \sum_{m=0}^{n}\left\|\varphi^{(n-m)}\right\|_{2, p+q}\left\|\psi^{(m)}\right\|_{2, p+q}\right)^{2} \\
& \leqq 2\|\varphi\|_{2, p+q}^{2}\|\psi\|_{2, p+q}^{2},
\end{aligned}
$$

where we used Proposition A.1, $\left\|\varphi^{(n)}\right\|_{2, p} \leqq\left\|A^{-1}\right\|\left\|^{q n}\right\| \varphi^{(n)} \|_{2, p+q}$ and Schwarz' inequality. $q$ was chosen such that $2^{-q} K \leqq 1 / 2$, i.e. independent of $\varphi$ and $\psi$. 
Acknowledgements. We have profited very much from helpful discussions with Professors S. Albeverio, M. Fukushima, M. Takeda, and Y. Yokoi. One of us (L.S.) gratefully acknowledges the kind hospitality of Professor T. Hida and the stimulating atmosphere of this Probability group at Nagoya University.

\section{References}

1. Albeverio, S., Hoegh-Krohn, R.: Dirichlet forms and diffusion processes on Rigged Hilbert spaces. Z. Wahrsch. 40, 1 (1977)

2. Albeverio, S., Hoegh-Krohn, R., Streit, L.: Energy forms, Hamiltonians and distorted Brownian paths. J. Math. Phys. 18, 907 (1977)

3. Albeverio, A., Hoegh-Krohn, R., Streit, L.: Regularization of Hamiltonains and processes. J. Math. Phys. 21, 1634 (1980)

4. Albeverio, S., Fukushima, M., Karkowski, W., Streit, L.: Capacity and quantum mechanical tunneling. Commun. Math. Phys. 81, 501 (1981)

5. Fukushima, M.: Dirichlet forms and Markov processes. Amsterdam: Kodansha and NorthHolland 1980

6. Fukushima, M.: Energy forms and diffusion processes. In: Mathematics and Physics. Lectures on recent results. Vol. 1. Streit, L. (ed.). Singapore: World Scientific 1985

7. Hida, T.: Analysis of Brownian functionals. Carelton Mathematical Lecture Notes, No. 13 (1975)

8. Hida, T.: Brownian Motion. Berlin, Heidelberg, New York: Springer 1980

9. Hida, T., Kuo, H.-H., Potthoff, J., Streit, L.: White noise: an infinite dimensional calculus (in preparation)

10. Kato, T.: Perturbation theory for linear operators. Berlin, Heidelberg, New York: Springer 1966

11. Kubo, I., Takenaka, S.: Calculus on Gaussian white noise. II. Proc. Jpn. Acad. 56, Ser. A, 411 (1980)

12. Kubo, I., Yokoi, Y.: A remark on the space of testing random variables in the white noise calculus. Preprint (1987)

13. Kuo, H.-H.: Brownian functionals and applications. Acta Appl. Math. 1, 175 (1983)

14. Kusuoka, S.: Dirichlet forms and diffusion processes on Banach spaces. J. Fac. Sci. Univ. Tokyo 29, 79 (1982)

15. Potthoff, J.: On positive generalized functionals. J. Funct. Anal. 74, 81 (1987)

16. Potthoff, J.: White-noise approach to Malliavin's calculus. J. Funct. Anal. 71, 207 (1987)

17. Potthoff, J.: On Meyer's equivalence. TUB preprint (1986)

18. Simon, B.: The $P(\phi)_{2}$ Euclidean (quantum) field theory. Princeton, NJ: Princeton University Press 1974

19. Takeda, M.: On the uniqueness of the Markovian self adjoint extension of diffusion operators on infinite dimensional space. Osaka J. Math. 22, 733 (1985)

20. Takeda, M.: On the uniqueness of the Markovian self-adjoint extension. BiBoS preprint No. 73 (1985)

21. Yokoi, Y.: Positive generalized Brownian functionals (in preparation)

Communicated by H. Araki

Received June 30, 1987 
